\title{
Interactive Play and Learning for Children
}

\author{
Adrian Cheok, ${ }^{1,2}$ Hiroshi Ishii, ${ }^{3}$ Junichi Osada, ${ }^{4}$ \\ Owen Noel Newton Fernando, ${ }^{1}$ and Tim Merritt ${ }^{1}$ \\ ${ }^{1}$ Mixed Reality Lab, Interactive and Digital Media Institute (IDMI), National University of Singapore, \\ 21 Lower Kent Ridge Road, Singapore 119077 \\ ${ }^{2}$ Graduate School of Media Design, Keio University, Kanagawa-ken 223-8522, Japan \\ ${ }^{3}$ MIT Media Lab, School of Architecture and Planning, Massachusetts Institute of Technology, Cambridge, MA 02139-4307, USA \\ ${ }^{4}$ NEC Design, Ltd., Shinagawa-Ku, Tokyo 141-0031, Japan
}

Correspondence should be addressed to Adrian Cheok, adriancheok@mixedrealitylab.org

Received 24 November 2008; Accepted 24 November 2008

Copyright ( $\odot 2008$ Adrian Cheok et al. This is an open access article distributed under the Creative Commons Attribution License, which permits unrestricted use, distribution, and reproduction in any medium, provided the original work is properly cited.

\section{Introduction}

One of the most socially and culturally beneficial uses of human computer interaction research is enhancing play and learning for children. It is very important to understand the needs of children and craft visionary interactive systems designed to enhance education and entertainment. In this special issue, we can study papers that provide visions and glimpses into the future of human computer interaction for children's play and learning.

Since the research findings of Piaget [1], educators have valued the activity of play as an essential stage for the development of the human mind. Through trial and error and a "learn by doing approach," children internalize concepts of complex physics, abstract logic, and social rules among others. Caillois [2] described man as essentially an organism that requires play. The positive psychology approach would also agree that this is especially critical in promoting the human to not only develop but truly thrive. It is very important to understand the needs of children and craft visionary interactive systems designed to enhance education and entertainment and provide those experiences and activities which allow the child to emerge as an empowered learner with confidence and inspiration.

\section{Guide to the Special Issue}

The focus of this special issue is to provide visions and glimpses into the future of human computer interaction for children's play and learning. It also discusses achievements on different technical and theoretical development of interactive play and learning for children. The special issue received an overwhelming number of submissions from researchers around the world focusing on various topics ranging from user-centered design to advanced technical developments. Considering originality, technical competence, user evaluation, and balance of topics, thirteen papers were accepted for the special issue. If we look at all of the papers as one body of works, it makes sense to divide them into three chapters according to the main focus. There were four focused on designing for children with disabilities, five focused on methodological issues of design for children, and four focused on specific technologies focused on supporting children in learning through play activities.

\subsection{Design for Disabilities}

Apart from being a most worthy application of research, it is a very well accepted strategy to focus on the design for people with disabilities as a way to improve designs for all users. By designing for users with specific needs, there are usability issues and needs that emerge which face the designer which are then transferrable to all users regardless of their abilities.

The paper by van Rijn and Stappers presents guidelines for designers of interactive systems for children with autism. These were developed during their LINKX project in order to design a language-learning toy for autistic children. Theoretical and practical implications for each guideline are presented through the experiences of the prototype 
development process so that other designers can use them to inform their design decisions.

The paper by Tuominen et al. presents research of their PROAGENTS multimodal learning environment, which supports blind children in explorative learning experiences with astronomy. The system utilizes haptic and auditive interaction techniques in the proactive agents that support and guide children, deepen their explorations, and discover the central concepts and other phenomena.

The paper by Hengeveld et al. presents research focused on children with multiple disabilities (e.g., both a cognitive and a motor disability) and stimulating the adult-child communication to decrease functional limitations. LinguaBytes is presented, which stimulates language development through interactive and adaptive play and learning in an environment incorporating tangible objects and multimedia content. The research is based on interactive storytelling and anchored instruction. An outline of the development of the LinguaBytes play and learning environment from the earliest studies, up to the current prototype, CLICK-IT, is provided.

The paper by Jacob and Mythili has developed a textto-speech (TTS) system specifically targeting children of various abilities including the vocally disabled. Their system utilizes linguistic analysis instead of the signal processing techniques used in other existing concatenative TTS systems. The paper presents the results of a user study, informing their development and giving insights to the challenges and needs of such a system.

\subsection{Methodology}

The advancement of the methodological tools for the field of HCI research and for designers in general represents a fundamental way of sharing knowledge and the blueprints for successful outcomes. Papers in this section recognized the creativity of the child as a designer, user-centered design, and the development of new multimodal environments.

The paper by Nousiainen and Kankaanranta presents their research experiences in user-centered and participatory design while in collaboration with elementary school children in the design of learning environments, based on three projects and requirements gathering techniques. Through this research, issues related to design collaboration with children emerged, especially in terms of the children's feeling of ownership over the final outcome. The children's contribution yielded outcomes which were useful in informing the design of the user interface as well as the contents of the learning environments.

The paper by Abeele and Zaman presents theoretical framework and practical case for designing likeable interactive media applications for preschoolers. In this article, they have demonstrated how the uses and gratifications paradigm together with expectancy-value theory could fill in one of the blanks in designing likeable applications for preschoolers via the research project CuTI (Cuddle Toy Interface). The CuTI project aims at revealing those particular user gratifications and design attributes that are important to support playful behavior and fun activities of preschoolers in the home environment.
The paper entitled "Child-centered evaluation: broadening the child/designer dyad" by Pardo et al. discusses challenges in literal interpretation of user-centered design for children. They have explored the certain hard-to-reach or difficult-to-interpret situations such as evaluation of educational software intended to be used by children. The paper also argues that the added value that teachers can bring to the evaluation of educational software can improve the diagnostic power of the evaluation and its sensitivity to pedagogical issues.

The paper by Motoyoshi et al. proposes a novel strategy of designing play equipments and detailed explanation of the strategy through an example of a designing process of playing environment where the players' usage of the play equipments cannot be foreseen. This enables designers to interpret the playing environment as an information channel in terms of channel theory, which is also an appropriate loose constraint for designing a play environment.

The paper by Birchfield et al. introduces theoretical research from HCI and Education that reveals a convergence of trends focused on embodiment, multimodality, and composition. Authors argued that there is great potential for truly transformative work that aligns HCI and education research, and posit that there is an important opportunity to advance this effort through the full integration of the three themes into a theoretical and technological framework for learning.

\subsection{Specific Applications and Technology}

Four of the papers clearly introduced innovative uses of technology in the creation of interactive systems for learning and play. From the systems which inspire creativity to the systems which assist in the mastery of games of skill, there are lessons to be learned and inspiration to be drawn from these cutting edge systems.

The paper entitled "BlogWall: social and cultural interaction for children" by Cheok et al. is an extension of the existing text messaging to a new level of self-expression and public communication, combining visual art and poetry. By selecting proper elements, the application can be effectively used to build awareness among children about diverse cultural elements of humans as global beings while they interact and play with the system. The results of users' studies indicated that the application has a high level of social and engagement aspects.

The paper by Kerdvibulvech and Saito proposes a visionbased method for tracking guitar fingering position and actions by guitar players. A new framework for tracking colored finger markers is presented which integrates a Bayesian classifier into particle filters. The resulting application made from this system assists guitar learners by allowing them to automatically identify if they are using the correct chords required by the musical piece. This brings HCI research and applies engineering solutions to enhance the creativity and learning of guitar playing.

The paper by Uematsu and Saito presents visually enhanced sports entertainment applications: AR baseball presentation system and interactive AR bowling system. 
Main advantage of these applications is that they do not require any special device such as positioning sensors or a high-performance PC because applications can be run on the tabletop in the real $3 \mathrm{D}$ world only with a web camera and a handheld monitor connected to a PC. Demonstration showed that children really enjoyed these applications because their actions in the real world affect the virtual world.

The paper by Uchiyama and Saito presents a pool supporting system with a camera-mounted handheld display based on augmented reality technology. Main objective of this research is to estimate and analyze the distribution of balls and to present visual aids without special equipment such as a magnetic sensor or artificial markers. Children are able to capture a pool table from an arbitrary viewpoint and see supporting information and ball behavior which are drawn on the captured images through the display. Results of the experiment showed that the accuracy of estimated ball positions is enough for analysis of ball arrangement.

\title{
3. Conclusion
}

We appreciate the opportunity to gather together a fine collection of academic papers which present a picture of the current landscape of the design of interactive and digital systems supporting learning and play for children. We hope that this special issue will inspire the researchers of today and tomorrow to create a better world promoting the learning and play activities of children of all ages.

\author{
Adrian David Cheok \\ Hiroshi Ishii \\ Junichi Osada \\ Owen Noel Newton Fernando \\ Tim Merritt
}

\section{References}

[1] J. Piaget, The Construction of Reality in The Child, Basic Books, New York, NY, USA, 1954, translated by Margaret Cook.

[2] R. Caillois, Man, Play and Games, Free Press of Glencoe, New York, NY, USA, 1961. 

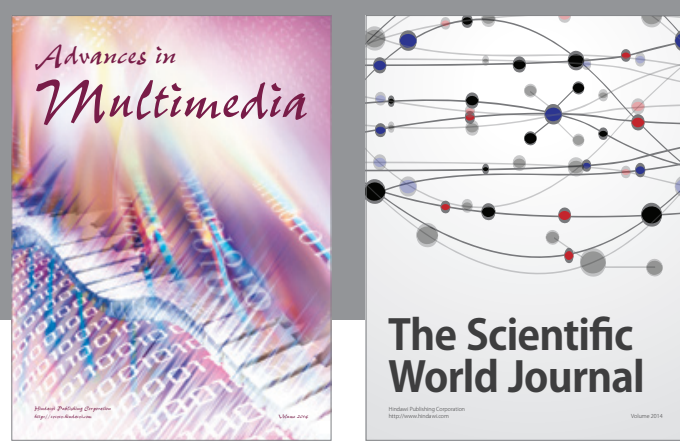

The Scientific World Journal
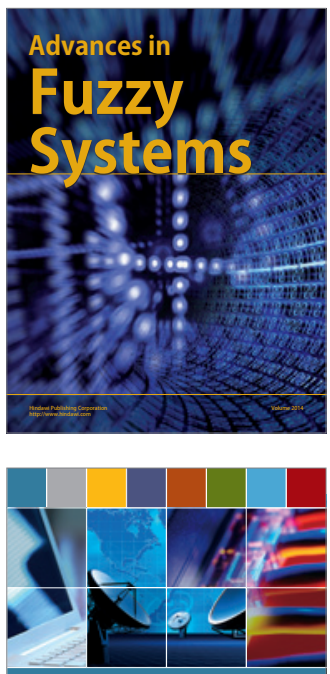

Computer Networks and Communications
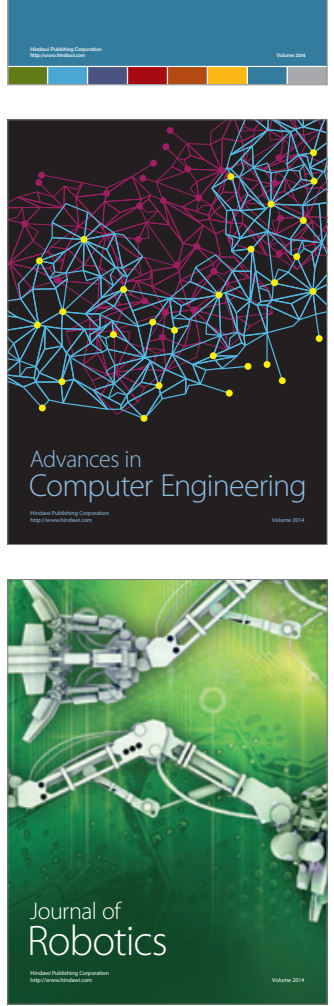
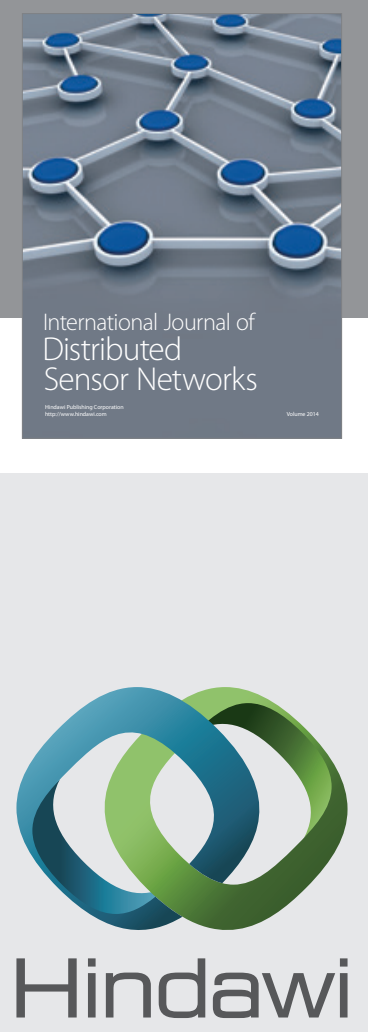

Submit your manuscripts at

http://www.hindawi.com
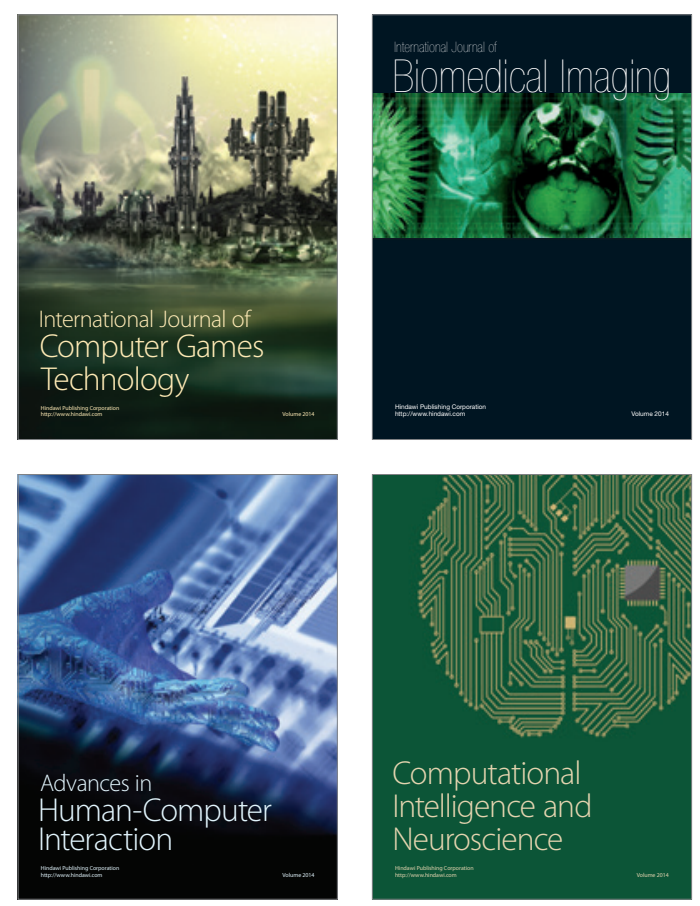
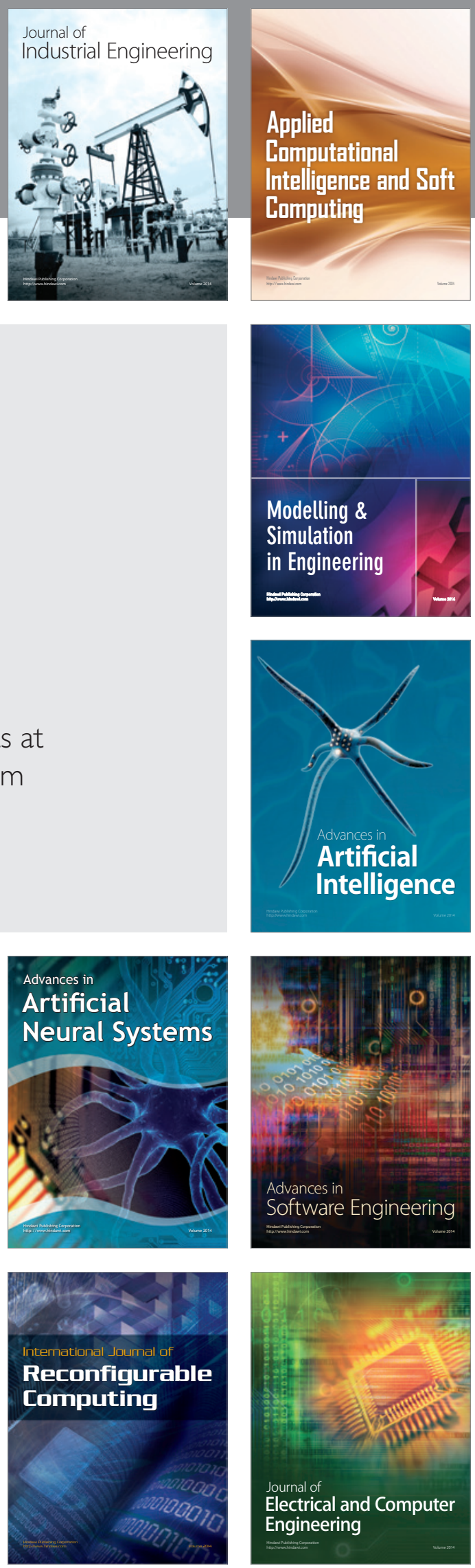